\title{
A NEW CODE FOR ORBIT RESPONSE MATRIX ANALYSIS*
}

\author{
Lingyun Yang ${ }^{\dagger}$, S.Y. Lee, Indiana University Cyclotron Facility, IN 47408, USA \\ Xiaobiao Huang, Stanford Linear Accelerator Center, CA \\ Boris Podobedov, BNL, Upton 11973, USA
}

\section{Abstract}

Orbit Response Matrix(ORM) method has been widely used for lattice modeling, and beam diagnostics, however the difficulty on convergence arises as the model becomes larger, more complicated or with nonlinear parameters brought in. A new code named YAOC(Yet Another Orbit Code) was developed based on a robust numerical method called scaled Levenberg-Marquardt algorithm, and its applications on various lattices are discussed here.

\section{INTRODUCTION}

Orbit Response Matrix, because of its direct connection to beam dynamics between two points in a storage ring, has been used for lattice modeling, and beam diagnostics $[6,2]$. But the difficulty on convergence arises as the model becomes larger, more complicated or has nonlinear parameters such as quadrupole strength brought in. YAOC was developed to improve convergence properties and to be more robust. Based on Scaled Levenberg-Marquardt algorithm, this code has resolved the coupling problems between parameters and gained excellent convergence in our thousands of simulations[2]. The applications on Fermilab Booster and Taiwan Photon Source design lattice is discussed in this paper. A recent experiment at VUV ring diagnosing the source of $60 \mathrm{~Hz}$ vertical orbit oscillation also proved its accuracy.

\section{ORBIT RESPONSE MATRIX}

Closed orbit shift observed at $s$ due to a small bump or a perturbation at $s_{0}$ is described by[3]

$$
\begin{aligned}
y_{c o}(s) & =\frac{\sqrt{\beta(s) \beta\left(s_{0}\right)}}{2 \sin \pi \nu} \cos \left(\pi \nu-\left|\phi(s)-\phi\left(s_{0}\right)\right|\right) \theta\left(s_{0}\right) \\
& \equiv G\left(s, s_{0}\right) \theta\left(s_{0}\right)
\end{aligned}
$$

where $\beta(\cdot)$ and and $\phi(\cdot)$ are the betatron amplitude functions and phases at source and observation locations, $\nu$ is betatron tune, $G\left(s, s_{0}\right)=d y_{c o}(s) / d \theta\left(s_{0}\right)$ is the Green function connecting a small bump $\theta\left(s_{0}\right)$ and an observed closed orbit shift at $y_{c o}(s)$. The closed orbit shift due to distributed bumps is a sum of all these effects: $y_{c o}(s)=$ $\sum_{i=0}^{n} G\left(s, s_{i}\right) \theta\left(s_{i}\right)$ For a storage ring with $m$ observation point, i.e. $m$ Beam Position Monitors(BPM), which can

\footnotetext{
* Work supported by grants from NSF PHY-0552389 and DOE DEFG02-92ER40747

†1y1@indiana.edu
}

05 Beam Dynamics and Electromagnetic Fields detect both horizontal and vertical displacement, the Green functions can form a matrix called Orbit Response Matrix:

$$
\left(\begin{array}{l}
x \\
z
\end{array}\right)_{2 m}=\left(\begin{array}{ll}
\mathbf{G}^{X X} & \mathbf{G}^{X Z} \\
\mathbf{G}^{Z X} & \mathbf{G}^{Z Z}
\end{array}\right)_{2 m \times 2 n}\left(\begin{array}{l}
\theta^{x} \\
\theta^{z}
\end{array}\right)_{2 n}
$$

where $n$ is the number of orbit bumps or perturbations, $\theta^{x}$ and $\theta^{z}$ are their horizontal and vertical bump strength, $x$ and $z$ are detected horizontal and vertical closed orbit shift, and $G^{\{\cdot\}\{\cdot\}}$ are matrices of Green functions, i.e. $G_{i j}^{X X}=d x_{i} / d \theta_{j}^{x}, G_{i j}^{Z Z}=d z_{i} / d \theta_{j}^{z}$. The off-diagonal submatrices, $G_{i j}^{Z X}=d z_{i} / d \theta_{j}^{x}$ and $G_{i j}^{X Z}=d x_{i} / d \theta_{j}^{z}$ would be zero, if no coupling presents between horizontal and vertical planes[2].

\section{MODELING AND OPTIMIZATION}

ORM usually has thousands of numbers. e.g. NSLS VUV ring with 24 BPMs and 16 orbit trim quads can have 1536 data points. Because of this large number of data, A lattice with variable parameters can be modeled on it, and the agreement between model and real running machine is described by a merit-function $\chi^{2}$ based on ORM data from measurement and lattice model:

$$
\begin{aligned}
\chi^{2}(p) \equiv & \sum_{i}^{N} f_{i}(p)^{2} \\
= & \sum_{i j}\left(\frac{\mathbf{G}_{i j}^{\{X, Z\}, \text { meas }}-\mathbf{G}_{i j}^{\{X, Z\}, \text { model }}}{\sigma_{\mathbf{G}_{i j}}}\right)^{2} \\
& +\sum_{x, z}\left(\frac{\nu_{x, z}^{\text {meas }}-\nu_{x, z}^{\text {model }}}{\sigma_{\nu_{x, z}}}\right)^{2} \\
& +\sum_{k}\left(\frac{\phi_{k}^{\{X, Z\}, \text { meas }}-\phi_{k}^{\{X, Z\}, \text { model }}}{\sigma_{\phi_{i j}}}\right)^{2}
\end{aligned}
$$

where $f$ is a vector composed of $G_{i j}^{\{\cdot\}\{\cdot\}}, \nu_{x, y}, \phi_{i j}$ and other possible observable quantities. $p$ is the variable parameters vector, which determines the $G_{i j}^{\{\cdot\}\{\cdot\} \text {,model }}$, $\nu_{x, z}^{\text {model }}$ and $\phi_{k}^{\{X, Z\}, \text { model }}$.

Although Newton's method with Sigular Value decomposition(SVD) can be used to minimize the figure-of-merit function $\chi^{2}[6]$, the convergence of fitting is very much limited within linear dependence of $\chi^{2}$ on orbit bump strength $\theta$, BPM tilt angle and gain. For a lattice model with nearly 10,000 ORM elements, and a few hundred variable parameters, the convergence indicator $\chi^{2} / N_{d}$, where $N_{d}$ is degree of freedom, is misleading due to a large denominator $N_{d}[2]$.

D01 Beam Optics - Lattices, Correction Schemes, Transport 
Scaled Levenberg-Marquardt(L-M) algorithm[5] is an effective and robust solution to nonlinear fitting problems with hundreds of variable parameters. Different from bump strength $\theta$ and BPM gain, the $K_{1}$ does not linearly change $G\left(s, s_{0}\right)$, therefore $y_{c o}(s)$ in Eq. (1). With scaled L-M algorithm, not only the linear parameters like orbit bump strength $\theta$, BPM gain factor, but also the nonlinear parameters such as quadrupole strength $K_{1}$ can be fitted correctly. The scaling property of L-M algorithm also makes it robust to avoid the betatron resonance.

The minimization of Eq. (3) has a general form as

$$
\min \{\|f+J p\|:\|D p\| \leq \Delta\}
$$

where $\|\cdot\|$ is length of vector, $J$ is any matrix of right dimension, $p$ is the parameter vector, and $D$ is scaling matrix, $\Delta$ can be regarded as the size of hyper-ellipsoid within which the solution is iteratively searched. Eq. (4) basically means using scaling matrix $D$ to searching for an optimum $p$, which minimize $\chi^{2}$, and $p$ is confined within a hyperellipsoid defined by $\Delta$. The iteration converges when arrives at a small enough $\Delta$.

Instead of solving a linear equation for $\delta p$ during iteration as in Newton's method[6], Scaled L-M algorithm, for example implemented in GSL[1], use a set of strategies to update $D$ and $\Delta$ to adaptively change $p$. This back-and-forth searching can effectively jump over betatron resonance[4,5].

\section{APPLICATIONS}

YAOC has been successfully applied on lattice simulations and beam diagnosis of Fermilab Booster, NSRRC design lattice, and NSLS VUV ring.

The design lattice is first input into YAOC, and together with a list of variable parameters, which can be BPM tilt angle, horizontal and vertical gain, quadrupole tilt angle, and strength $K_{1}$. Following the strategies of scaled L$\mathrm{M}$ algorithm, YAOC will search for an optimum set of these parameters making their ORM as close as possible to the measured ORM. The lattice function routines are adapted from Elegant to speed up the first derivatives $\partial \chi^{2} / \partial p_{i}$ and the second derivatives, i.e. the Jacobian Ma$\operatorname{trix} J_{i j}=\partial^{2} \chi^{2} / \partial p_{i} \partial p_{j}$.

Fermilab Booster lattice was simulated extensively on thousands of lattice cases with different random errors. In each lattice case, a small error $\Delta K_{1}$ was added to every combined function dipoles(also called quadrupoles in my lattice modeling) which has a nominal $K_{1}=$ $\pm 0.05 m^{-2}$, and new Orbit Response Matrix was generated as experiment data. YAOC was applied to fit for these $\Delta K_{1}$, and Fig. 1 shows a typical result of our fitting, where fitted $\Delta K_{1} \mathrm{~s}$ are the same as those we used to generate the input ORM data. A statistics of YAOC's performance is in Fig. 1(b), where the errors of quadrupole strength have a uniform distribution with $\Delta K_{1} \in\left[-10 \% K_{1},+10 \% K_{1}\right]$. It shows good agreement 05 Beam Dynamics and Electromagnetic Fields

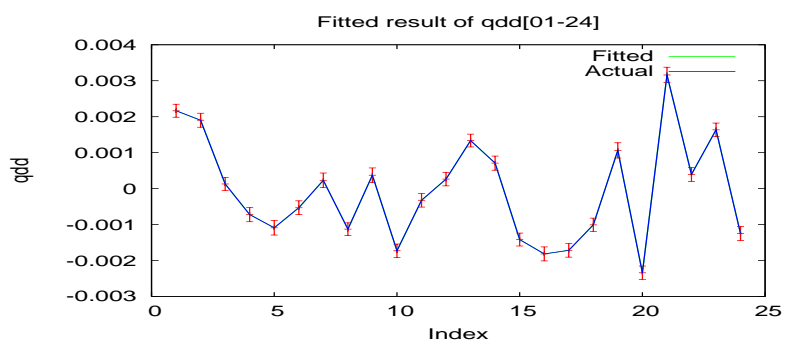

(a) One example of fitted results

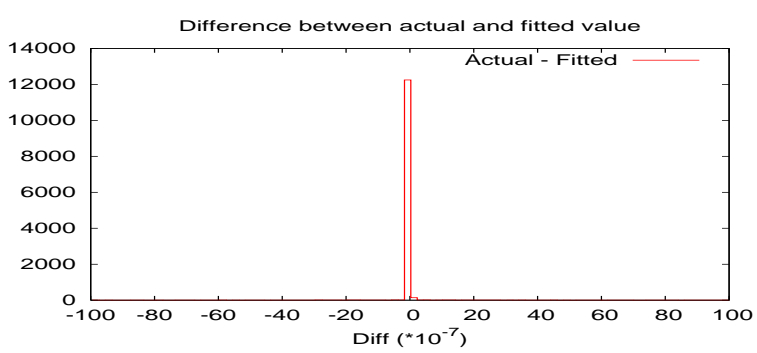

(b) Statistics of fitted results

Figure 1: Simulation of Fermilab Booster quadrupole errors

between fitted and actual quadrupole strength, and the difference are always less than $1 \times 10^{-5} \mathrm{~m}^{-2}$ which is only $0.02 \%$ of nominal $K_{1}$ value. Over 3000 lattices are studied, with different distribution of random errors, and the convergence is almost guaranteed in Fermilab Booster simulations when only quadrupole and BPM errors are considered.

BPM noise is also considered in simulations. In Fig. 2, $\pm 10 \mu \mathrm{m}$ amplitude noise is added to the closed orbit shift, The difference of quadrupole strength between YAOC output and actual lattice is less than $1 \%$ of the nominal $K_{1}$.

YAOC is also applied to Taiwan Photon Source design lattice, which has 24 superperiods, 600 parameters including quadrupoles and BPMs errors, and 112898 terms in $\chi^{2}$. The distortion of betatron amplitude functions due to quadrupole errors are shown in Fig. 3. Starting from design lattice YACO successfully reproduced the $b e t a_{x, y}$ almost

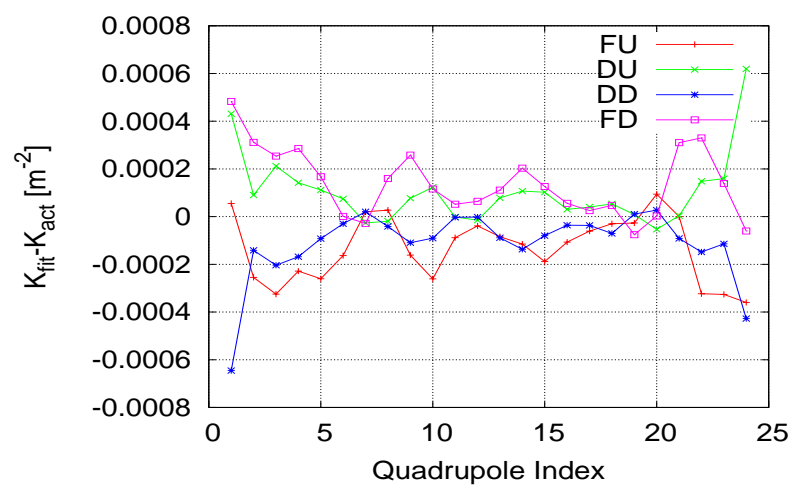

Figure 2: A result with BPM noise $10 \mu \mathrm{m}$

D01 Beam Optics - Lattices, Correction Schemes, Transport 
same as the simulated measurement. The fitted and "measured" $\beta_{x}$ are overlapped in Fig. 3. The design lattice can be restored by comparing the quadrupole strength output of YAOC with design values.

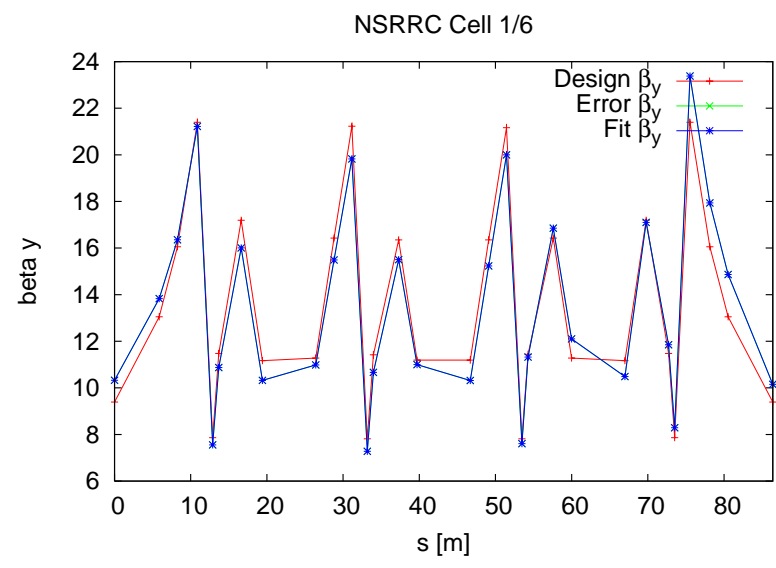

Figure 3: Lattice Modeling of NSRRC.

A recent study at NSLS VUV also shows the ability of YAOC in beam diagnosis. We have used YAOC to locate the source of orbit vibration. The orbit vibration data is dumped from BPM buffer, and a model with one virtual bumper was built in YAOC. After comparing the single column of ORM due to this virtual bumper and the BPM buffer data, we find out the most possible location is at BUISH(Booster UV ring Injection Septum) indicated by the minimum $\chi^{2}$ in Fig. 4 .

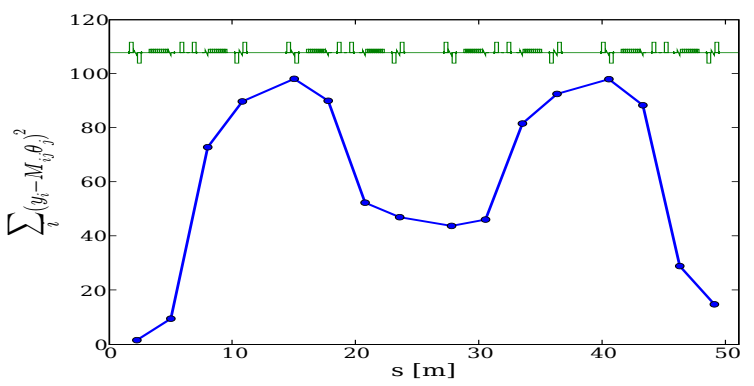

Figure 4: YAOC was used to find the source of vertical orbit vibration. $\chi^{2}$ of orbit vibration indicates the source is located near injection.

After turning off the BUISH power supply, the vertical orbit perturbation disappeared. Fig. 5 compares the orbit vibration with BUISH on and off at two BPMs. Due to phase advance between source and observation point, the vibration amplitude is different at each BPM. A proper phase advance may make the sin term in Green function be zero, therefore the orbit at that BPM is not affected by perturbation at this particular source point. This is the case for PUE17 shown in Fig. 5. After turning off BUISH, orbit vibration at $24 \mathrm{BPMs}$ are all below $10 \mu \mathrm{m}$. It confirms that the perturbation source of vertical orbit vibration is from 05 Beam Dynamics and Electromagnetic Fields
BUISH.
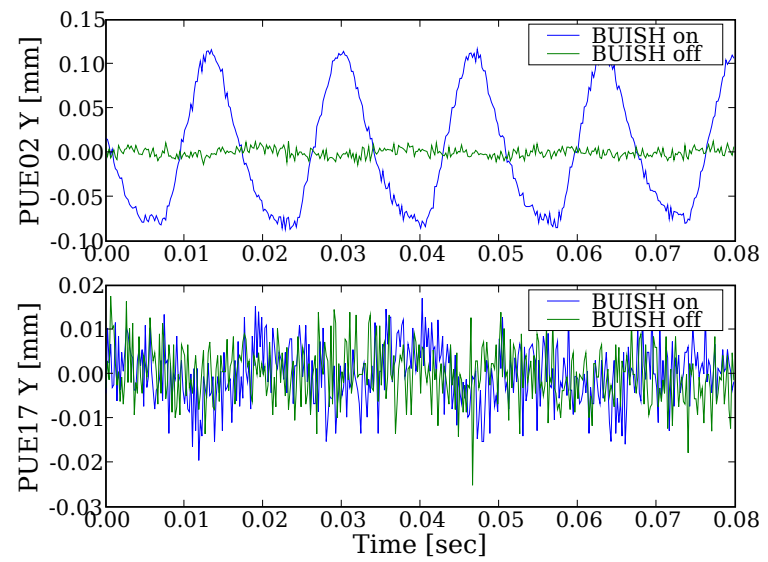

Figure 5: Vertical Orbit Vibration with BUISH(Booster UV ring Injection Septum) on/off(feedback is off for both cases).

\section{CONCLUSION}

YAOC is an orbit optimization code based on scaled Levenberg-Marquardt method. It provides better convergence properties, and resolves parameter coupling problems. It is also immune from a reasonable level of BPM noise. Applications of the new code has been successful on Fermilab Booster, Taiwan Photon Source design lattice, VUV ring.

\section{ACKNOWLEDGEMENTS}

We would like to thank VUV operators for their help on measurements, Stephen Kramer and James A. Safranek for helpful discussion with them.

\section{REFERENCES}

[1] GSL: GNU scientific library.

[2] Xiaobiao Huang, Beam diagnosis and lattice modeling of the fermilab booster, Ph.D. thesis, Indiana University at Bloomington, August 2005.

[3] S. Y. Lee, Accelerator physics, 2 ed., World Scientific, 2004.

[4] D.W. Marquardt, An algorithm for least-dquares estimation of nonlinear parameters, Journal of the Society for Industrial and Applied Mathematics 11 (1963), 431-441.

[5] J. J. More, The levenberg-marquardt algorithm: Implementation and theory, Lecture Notes in Mathematics (G. Watson, ed.), vol. 630, Springer, 1978, p. 105.

[6] J. Safranek, Beam-based lattice diagnostics, Proceedings of the Joint US-CERN-JAPAN-RUSSIA School on Particle Accelerators, Beam Measurement (E.Perevedentsev Si.Kurokawa, S.Y.Lee and S.Turner, eds.), World Scientific, 1998.

D01 Beam Optics - Lattices, Correction Schemes, Transport 